\title{
The correlation of metrics in complex networks with applications in functional brain networks
}

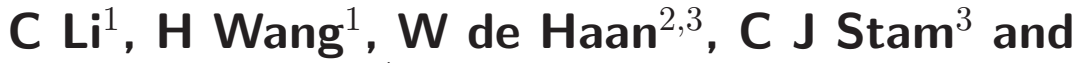 \\ P Van Mieghem ${ }^{1}$ \\ ${ }^{1}$ Faculty of Electrical Engineering, Mathematics and Computer Science, \\ Delft University of Technology, 2628CD Delft, The Netherlands \\ 2 Alzheimer Center, Department of Neurology, VU University Medical Center, \\ 1081HV Amsterdam, The Netherlands \\ ${ }^{3}$ Department of Clinical Neurophysiology, VU University Medical Center, \\ 1081HV Amsterdam, The Netherlands \\ E-mail: Cong.Li@tudelft.nl, H.Wang@tudelft.nl,w.dehaan@vumc.nl, \\ CJ.Stam@vumc.nl and P.F.A.VanMieghem@tudelft.nl
}

Received 5 October 2011

Accepted 1 November 2011

Published 25 November 2011

Online at stacks.iop.org/JSTAT/2011/P11018

doi:10.1088/1742-5468/2011/11/P11018

\begin{abstract}
An increasing number of network metrics have been applied in network analysis. If metric relations were known better, we could more effectively characterize networks by a small set of metrics to discover the association between network properties/metrics and network functioning. In this paper, we investigate the linear correlation coefficients between widely studied network metrics in three network models (Bárabasi-Albert graphs, Erdös-Rényi random graphs and Watts-Strogatz small-world graphs) as well as in functional brain networks of healthy subjects. The metric correlations, which we have observed and theoretically explained, motivate us to propose a small representative set of metrics by including only one metric from each subset of mutually strongly dependent metrics. The following contributions are considered important. (a) A network with a given degree distribution can indeed be characterized by a small representative set of metrics. (b) Unweighted networks, which are obtained from weighted functional brain networks with a fixed threshold, and ErdösRényi random graphs follow a similar degree distribution. Moreover, their metric correlations and the resultant representative metrics are similar as well. This verifies the influence of degree distribution on metric correlations. (c) Most metric correlations can be explained analytically. (d) Interestingly, the most studied
\end{abstract}


The correlation of metrics in complex networks with applications in functional brain networks

metrics so far, the average shortest path length and the clustering coefficient, are strongly correlated and, thus, redundant. Whereas spectral metrics, though only studied recently in the context of complex networks, seem to be essential in network characterizations. This representative set of metrics tends to both sufficiently and effectively characterize networks with a given degree distribution. In the study of a specific network, however, we have to at least consider the representative set so that important network properties will not be neglected.

Keywords: neuronal networks (experiment), network dynamics, random graphs, networks, computational neuroscience

\section{Contents}

1. Introduction 2

2. Definition of network metrics 5

3. Analytic relations between network metrics 8

3.1. General relations . . . . . . . . . . . . . . . . . 8

3.2. Analytic relations in Erdös-Rényi random graphs . . . . . . . . . . . . . . 9

3.3. Analytic relations in Bárabasi-Albert graphs . . . . . . . . . . . . . . . . 10

4. Metric correlations in network models 11

4.1. Erdös-Rényi random graphs . . . . . . . . . . . . . . . . . . . . . . 12

4.2. Bárabasi-Albert graphs . . . . . . . . . . . . . . . . . . . . 14

4.3. Watts-Strogatz small-world graphs . . . . . . . . . . . . . . 15

5. Metric correlations in functional brain networks $\quad 17$

5.1. Metric correlations in unweighted functional brain networks transformed with fixed threshold $T \ldots \ldots \ldots \ldots$. . . . . . . . . . . . 18

5.2. Metric correlations in unweighted functional brain networks transformed with fixed link density $p \ldots \ldots$. . . . . . . . . . . . . . . 20

6. Conclusion 20

$\begin{array}{ll}\text { Acknowledgments } & 22\end{array}$

Appendix A. Metric correlations in Erdös-Rényi random graphs (figures A.1 and A.2)

Appendix B. Metric correlations in Bárabasi-Albert graphs (figure B.1) 24

Appendix C. Metric correlations in Watts-Strogatz small-world graphs (figure C.1)

\section{Introduction}

After about a decade of extensive research on complex networks, numerous metrics have been introduced to quantify different features of complex networks $[12,40]$. The computation complexity of network metrics can be high. Actually, network metrics can 
be strongly correlated in a certain type of graphs such as power-law graphs, indicating redundancy among them. On the other hand, each metric only partially captures the properties of a network. It would be helpful if it is possible to define a small representative set of network metrics that effectively characterize a given type of networks. Understanding the relations between network metrics is essential, in general, for complex network studies. In this paper, we take neuroscience as an illustration.

Network science has recently been applied to neuroscience to understand the effect of the network structure on its functioning. The average shortest path length in the human functional brain network was shown to be negatively correlated with IQ [26, 45]. The small-world pattern and modularity tend to disappear in the brain networks of patient groups with, for example, brain tumors, epilepsy or Alzheimer's [7,9]. Wellstudied metrics like degree diversity $(\kappa)$, assortativity $\left(\rho_{D}\right)$, clustering coefficient $\left(C_{G}\right)$, average hopcount $(E[H])$, global efficiency $(E[1 / H])$, spectral radius $\left(\lambda_{1}\right)$, effective graph resistance $\left(R_{G}\right)$, algebraic connectivity $\left(\mu_{N-1}\right)$ and ratio of $\mu_{1} / \mu_{N-1}$, have been applied to functional brain networks [40]. Is it redundant to consider these widely studied metrics to relate network property/metric to network functioning? Which set of metrics at least have to be considered? The understanding of the relation between network properties/metrics enables neuroscientists to discover the most relevant topological features/metrics that may characterize a certain brain disease or function [41].

This work investigates the correlations between network metrics, aiming to identify a small representative set of metrics by including only one metric from each subset of mutually strongly dependent metrics. Metric correlation was studied via the linear correlation coefficient between network metrics in real-world networks in [21]. However, that approach did not address the following challenges. First, the correlation between network metrics is topology-dependent. Consider, for example, the correlation between the average shortest path length ${ }^{4} E[H]$ and the number of nodes $N$. The average shortest path length $E[H]$ is independent of the size $N$ in the class of dense ErdösRényi random graphs but is positively correlated with $N$ in $D$-dimensional lattices by $E[H] \sim\left(N^{1 / D}(D / 3)\right)$. Thus, the correlation between two metrics can be different in different types of networks. In other words, the representative set of metrics can be different for different types of networks. Second, most network metrics are correlated with the number of nodes $N$ (or even the number of links $L$ ) of a network. This introduces the difficulties in comparing networks $[4,50]$. When the set of networks, like the set of real-world networks studied in [21] are of different size $N$, two metrics may seem to be strongly correlated, simply because they are both correlated with the size $N$. We approach these difficulties in three steps. (i) We consider three network models: the Erdös-Rényi random graphs ${ }^{5}$ [18] with a binomial degree distribution, the Bárabasi-Albert graphs ${ }^{6}$ [3] with a power-law degree distribution and the Watts-Strogatz small-world graphs ${ }^{7}$ [52]

\footnotetext{
${ }^{4}$ The shortest path length between a node pair is the number of links contained in the shortest path between the node pair. The average shortest path length $E[H]$ is the average over all node pairs.

${ }^{5}$ An Erdös-Rényi random graph can be generated from a set of $N$ nodes by randomly assigning a link with probability $p$ to each pair of nodes.

${ }^{6}$ A Bárabasi-Albert graph starts with $m$ nodes. At every time step, we add a new node with $m$ links that connect the new node to $m$ different nodes already present in the graph. The probability that a new node will be connected to node $i$ in step $t$ is proportional to the degree $d_{i}(t)$ of that node. This is referred to as preferential attachment.
} 
where most nodes have the same degree. This allows us to understand the influence of the degree distribution on metric correlations. Although other network properties may as well influence metric correlations, we start with the degree distribution since it is the most studied and usually the easiest to obtain in most complex networks. (ii) We consider metric correlations in the instances of each model with a given size $N$ and a given link density $p$. Initial results have been discussed in [12]. Here, we further explore how the metric correlations change with network parameters $N$ and $p$ to obtain the metric correlation pattern in each network model. (iii) The metric correlations are explored in the functional brain network of healthy subjects, which have the same network size $N$. So far, metric relations have been mainly studied via the linear correlation coefficient $[12,21,27]$, as in this paper. We do deem studies on other correlation measures, such as partial correlations and multivariate analysis $[15,27]$, which may lead to different observations, as valuable further work.

Section 2 introduces network metrics that we explore. The linear correlation coefficients between network metrics are computed in a large number of network instances of each model with various parameters in section 4 . The metric correlation patterns in network models as well as the corresponding representative sets of metrics are considered important contributions of this paper. Surprisingly, the large set of metrics that we considered can be sufficiently represented by a small number of metrics in Erdös-Rényi random graphs, Bárabasi-Albert graphs and Watts-Strogatz small-world graphs. The analytic relations between network metrics, presented in section 3, support the correlations discovered via numerical experiments in section 4.

Finally, we study metric correlations in the functional brain networks of healthy subjects in section 5. We discuss how to derive unweighted functional brain networks via fixed threshold or via fixed average degree [50]. First, with the fixed threshold, the unweighted functional brain networks are shown to follow approximately binomial degree distribution. Interestingly, the metric correlation pattern in the studied functional brain networks is consistent with what we found in the Erdös-Rényi random graph model. Second, with the fixed average degree or link density, the degree distribution of unweighted functional brain networks has a heavy tail. The metric correlation patterns of these two types of unweighted functional brain networks are different whilst their degree distributions differ.

Our results suggest that (a) the representative set of network metrics can indeed be smaller than the originally considered set; (b) the average distance and the clustering coefficient, the most studied metrics so far especially in neuroscience, are strongly correlated and, thus, redundant; (c) spectral metrics ${ }^{8}$ are only studied recently in the context of complex networks. However, at least one spectral metric appears in the representative set, suggesting the importance of spectral metrics in network characterizations. When we study a class of graphs with a given degree distribution, these networks can be possibly characterized by a small representative set of metrics instead of by the originally considered set. However, in the study of a specific complex network, the representative set at least has to be considered so as not to neglect any

${ }^{7}$ A Watts-Strogatz small-world graph can be generated from a ring lattice with $N$ nodes and $k$ edges per node, by rewiring each link at random with probability $p$.

${ }^{8}$ Spectral metrics are those involving in the eigenvalue computations, such as spectral radius $\left(\lambda_{1}\right)$, effective graph resistance $\left(R_{G}\right)$, algebraic connectivity $\left(\mu_{N-1}\right)$ and ratio of $\mu_{1} / \mu_{N-1}$. 
important network properties. In empirical network studies, we probably do not know the true underlying topology but partial network properties. Our understanding of the dependency of metric correlation patterns on network properties opens up the possibility to use metric correlation as a topology diagnostic for real networks.

\section{Definition of network metrics}

In this section, we introduce the network metrics that are widely studied in the literature, from classical structural metrics (from local metrics like degree diversity to global metrics, e.g. the average hopcount) to spectral metrics (eigenvalue-related metrics). The correlation between these metrics will be studied analytically in section 3 and experimentally in section 4 .

Let $G=(\mathcal{N}, \mathcal{L})$ be a graph, where the number of nodes is denoted by $N=|\mathcal{N}|$ and the number of links is represented by $L=|\mathcal{L}|$. The graph $G$ can be represented by a $N \times N$ adjacency matrix $A$, consisting of elements $a_{i j}$ that are either one or zero, depending on whether there is a link between nodes $i$ and $j$. The Laplacian matrix of $G$ is an $N \times N$ matrix $Q=\Delta-A$, where $\Delta=\operatorname{diag}\left(d_{i}\right)$ and $d_{i}$ is the degree of node $i \in \mathcal{N}$.

- Degree diversity $\kappa$.

The degree diversity $\kappa$ is defined [6] as

$$
\kappa=\frac{E\left[D^{2}\right]}{E[D]}=\frac{\operatorname{Var}[D]+E[D]^{2}}{E[D]}
$$

Chung et al [10] found that the degree diversity approximates the largest adjacency eigenvalue $\lambda_{1}$ in Erdös-Rényi random graphs, if $\kappa>\sqrt{d_{\max }} \ln N\left(d_{\max }\right.$ is the maximum degree). Scale-free networks, where $\operatorname{Var}[D] \rightarrow \infty$ as $N \rightarrow \infty$, are characterized by $\kappa \rightarrow \infty$, whereas regular networks, where $\operatorname{Var}[D]=0$, have $\kappa=E[D]$. Properties of dynamic processes on networks, such as the synchronization threshold in the mean-field theory of coupled oscillators in networks [38], the network percolation [11] and the epidemic thresholds [31], have all been stated to be related to $\kappa=E\left[D^{2}\right] / E[D]$, approximately.

- Assortativity $\rho_{D}$.

'Mixing' in complex networks [34] refers to the tendency of network nodes to connect preferentially to other nodes with either similar or opposite properties. The mixing of the degree is computed via the degree correlation of connected node pairs, called assortativity [48]:

$$
\rho_{D}=1-\frac{\sum_{i \sim j}\left(d_{i}-d_{j}\right)^{2}}{\sum_{i=1}^{N} d_{i}^{3}-(1 / 2 L)\left(\sum_{i=1}^{N} d_{i}^{2}\right)^{2}} .
$$

Networks where high-degree nodes preferentially connect to other high-degree nodes, are assortative in the degree correlation $\left(\rho_{D}>0\right)$, whereas networks where high-degree nodes connect to low-degree nodes, are disassortative $\left(\rho_{D}<0\right)$. Van Mieghem et al $[48,49]$ have reformulated the assortativity as follows:

$$
\rho_{D}=\frac{N_{1} N_{3}-N_{2}^{2}}{N_{1} \sum_{i=1}^{N} d_{i}^{3}-N_{2}^{2}}
$$


where $N_{k}=u^{T} A^{k} u$ is the total number of walks with $k$ hops. Newman [33] found that technological and biological networks are disassortative while social networks are assortative. The functional brain networks determined from EEG have also been found to be assortative [13].

- Clustering coefficient $C_{G}$.

Two different clustering coefficients are frequently used. The first definition of clustering coefficient $[11,25,46] C_{G}$ of a graph is the average clustering coefficient of nodes whose degree is larger than 1 , given as

$$
C_{G}=\frac{1}{N-\left|\mathcal{N}^{(1)}\right|} \sum_{v \in \mathcal{N}_{-\mathcal{N}^{(1)}}} c_{G}(v)
$$

where $\mathcal{N}$ is the set of all nodes and $\mathcal{N}^{(1)}$ is the set of degree 1 nodes. The clustering coefficient of a node $c_{G}(v)$ characterizes the density of connections in the environment of a node $v$ and is defined as the ratio of the number of links $y$ connecting the $d_{v}>1$ neighbors of $v$ over the total possible $d_{v}\left(d_{v}-1\right) / 2$, thus $c_{G}(v)=2 y / d_{v}\left(d_{v}-1\right)$.

The second one is based on the following definition for undirected unweighted networks [12]:

$$
C_{G}=\frac{3 \mathbf{\Delta}_{G}}{N_{\Lambda}}
$$

where $\boldsymbol{\Delta}_{G}$ is the number of triangles in the network and $N_{\Lambda}=\sum_{i=1}^{N}\left(\begin{array}{c}d_{i} \\ 2\end{array}\right)$ is the number of connected triples.

The difference between the two definitions is that equation (4) is the average of the connection density among the neighbors of each node, while equation (5) is the average of the probability that a triangle is formed upon each triple in the network. In this paper, we consider the effect of degree distribution, so we use the definition equation (4) to calculate the clustering coefficient to each node.

- Average hopcount $E[H]$ and global efficiency $E[1 / H]$.

The hopcount $H_{i j}$ is the number of links or hops in the shortest path between node $i$ and node $j$. The maximal hopcount $H_{\max }$ among all node pairs is the diameter of a graph. If the average hopcount of a network approximates that of the corresponding Erdös-Rényi random graph with the same number $N$ of nodes and link density $p\left(E\left[H_{G}\right] \approx E\left[H_{G_{p}(N)}\right]\right)$, and the clustering coefficient always $C_{G}>C_{G_{p}(N)}$, then the network possesses the smallworld property.

When a network is disconnected, the shortest paths between some node pairs are infinite, so the average hopcount of the network cannot be computed. In this situation, we compute the average reciprocal hopcount $E[1 / H]$, which is called the global efficiency and widely studied in neuroscience $[8,25]$. In addition to the global efficiency in [25], a local efficiency is defined as $E_{\mathrm{loc}}=1 / N \sum_{i \subset G} E\left(G_{i}\right)$, where $G_{i}$ is the subgraph of the neighbors of $i$. The local efficiency plays a role similar to the first definition of clustering coefficient $C_{G}$. As we have included the first definition of the clustering coefficient in a metric set, thus the local efficiency is not taken into account in this paper.

- Spectral radius (the largest adjacency eigenvalue) $\lambda_{1}$. 
We denote the set of eigenvalues of the adjacency matrix $A$ as $\lambda_{N} \leq \lambda_{N-1} \leq \cdots \leq \lambda_{1}$, where the largest eigenvalue $\lambda_{1}$ is called the spectral radius. The eigenvalues of the adjacency matrix are real, while the eigenvalues of the Laplacian matrix are real and nonnegative [28]. The largest eigenvalue $\lambda_{1}$ is a powerful character of dynamic processes on networks such as virus spreading and synchronization processes [38]. The inverse of the largest eigenvalue $\lambda_{1}$ characterizes the threshold of the phase transition, which specifies the onset of a remaining fraction of infected nodes and of locked oscillators, respectively, of both virus spread [47] and synchronization of coupled oscillators [37] in networks. Restrepo et al [38] discovered that $\lambda_{1}$ can be approximated by $N_{3} / N_{2}$, where $N_{k}$ is the total numbers of walks with $k$ hops. Recently, Van Mieghem et al [48] proved that $N_{3} / N_{2}$ is a lower bound of the largest adjacency eigenvalue $\lambda_{1}$. Both motivate us to consider $N_{3} / N_{2}$ as a potentially important network metric.

- Effective graph resistance $R_{G}$.

The effective graph resistance (which is also called the Kirchhoff index) originated from the field of electric circuit analysis [17,23]. Assuming a network as an electrical circuit where the resistance of each link is 1, the effective graph resistance is defined as the accumulated effective resistance between all pairs of vertices. It measures the ease of communication in a graph $[17,20]$. The equivalent spectral expression for the effective graph resistance is [49]

$$
R_{G}=N \sum_{k=1}^{N-1} \frac{1}{\mu_{k}}
$$

where $\mu_{k}$ is the $k$ th largest eigenvalue of the Laplacian matrix $Q$.

- Algebraic connectivity $\mu_{N-1}$.

The eigenvalues of the Laplacian matrix $Q$ are ordered as $0=\mu_{N} \leq \mu_{N-1} \leq \cdots \leq \mu_{1}$ and $\mu_{N-1}>0$ if and only if the graph $G$ is connected. The second smallest eigenvalue $\mu_{N-1}$ of $Q$ is called the algebraic connectivity. It was first studied by Fiedler [19]. A large value of algebraic connectivity characterizes strong network robustness regarding, for example, (a) the difficulty to cut the network into separated subparts [22] and (b) enhanced synchronizability and fast convergence [16,51].

- Ratio $\mu_{1} / \mu_{N-1}$.

The ratio of the largest eigenvalue $\mu_{1}$ and the second smallest eigenvalue $\mu_{N-1}$ of the Laplacian is often claimed as an index of synchronizability of a graph [44]. The synchronizability mainly indicates whether the synchronized state of a dynamic on a graph will be stable for a sufficiently large range of parameters of the dynamic process $[4,35]$. The larger the ratio is, the more difficult it is to synchronize the oscillators and vice versa [4]. The ratio is also referred to as the 'paradox of heterogeneity'. It shows that (unweighted, undirected) networks with a more homogeneous degree distribution synchronize more easily than networks with a more heterogeneous degree distribution [44]. In [49], Van Mieghem has explained that $\mu_{1}>D_{\max }$ and $\mu_{N-1} \leq D_{\min }$. Hence, $\mu_{1} / \mu_{N-1}>D_{\max } / D_{\min }$, implying that the ratio $\mu_{1} / \mu_{N-1}$ is larger for heterogeneous networks because $D_{\max } / D_{\min }$ is larger, while homogeneous networks have smaller ratios $\mu_{1} / \mu_{N-1}$. 
The correlation of metrics in complex networks with applications in functional brain networks

\section{Analytic relations between network metrics}

In this section, we will analytically derive relations between the network metrics introduced in section 2. Relations that have been proved in the literature will be presented as well. These analytic relations partially explain the observations of numerical experiments in section 4 .

\subsection{General relations}

Lemma 1. In any connected graph, for $N>1$, the effective graph resistance $R_{G}$ obeys

$$
\frac{R_{G}}{(N-1)^{2}} \geq \frac{1}{E[D]} \geq \frac{1}{\lambda_{1}}
$$

Proof. From [49 p 68], the sum of Laplacian eigenvalues equals

$$
\sum_{j=1}^{N-1} \mu_{k}=2 L
$$

so that, for any graph with $L>0$,

$$
\frac{1}{\sum_{j=1}^{N-1} \mu_{k}}=\frac{1}{2 L} \text {. }
$$

Jensen's inequality states that, if $f(x)$ is a convex function (see [46 section 5.2]),

$$
f(E[X]) \leq E[f(X)] .
$$

Since $f(x)=1 / x$ is convex when $x>0$, and nice in a connected graph, $\mu_{k}>0$, for $1 \leq k \leq N-1$, application of (9) to the left-hand side of (8) yields

$$
\frac{1}{(1 /(N-1)) \sum_{j=1}^{N-1} \mu_{k}} \leq \frac{1}{N-1} \sum_{j=1}^{N-1} \frac{1}{\mu_{k}},
$$

from which we obtain

$$
\sum_{j=1}^{N-1} \frac{1}{\mu_{k}} \geq \frac{(N-1)^{2}}{2 L}
$$

invoking the definition (6) of the effective graph resistance:

$$
\frac{R_{G}}{(N-1)^{2}} \geq \frac{N}{2 L}=\frac{1}{E[D]} \text {. }
$$

We note that (11) is another derivation of the inequality (7.25) in [49]. Using the classical bound [49] of the spectral radius $\lambda_{1} \geq E[D]$, we arrive by combining $1 / \lambda_{1} \leq 1 / E[D]$ and (11) at (7).

Inequality (7) supports the negative correlation between the effective graph resistance and the spectral radius, if the inequality is close to an equality.

Relation 1. For any connected graph

$$
\lambda_{1} \geq \frac{N_{3}}{N_{2}} .
$$

Proof. See [48]. 
Restrepo et al [38] have shown that the spectral radius $\lambda_{1}$ can be approximated by $N_{3} / N_{2}$. Hence, the inequality (12), proves the strong correlation $\rho\left(\lambda_{1}, N_{3} / N_{2}\right) \approx 1$. The equality occurs in regular graphs, so the more irregular a graph is, the worse is (12).

Relation 2. In any connected graph

$$
\frac{\mu_{1}}{\mu_{N-1}}=\frac{1}{\mu_{N-1}} \mu_{1}>\frac{1}{\mu_{N-1}}
$$

since $\mu_{1} \geq(N /(N-1)) D_{\max }>1$ (see [49]).

The inequality (13) supports the strong negative correlation between $\mu_{1} / \mu_{N-1}$ and $\mu_{N-1}$.

Relation 3. It is immediate from Jensen's inequality (9) that $1 / E[H] \leq E[1 / H]$.

Hence, the average hopcount $E[H]$ is negatively correlated with the global efficiency $E[1 / H]$.

Relation 4. As proved in [49 p 207]

$$
R_{G} \leq\left(\begin{array}{c}
N \\
2
\end{array}\right) E[H]
$$

The inequality (14) supports the positive correlation between the effective graph resistance and the average hopcount.

Relation 5. In any Erdös-Rényi random graph or approximately in Bárabasi-Albert graphs

$$
\lambda_{1} \geq \kappa
$$

Proof. The assortativity of an Erdös-Rényi random graph equals to 0, as proved in $[33,48]$. Via (3), we have

$$
N_{2}^{2}=N_{1} N_{3}
$$

where $N_{k}=u^{T} A^{k} u$ are the total numbers of walks with $k$ hops. Using

$$
E[D]=\frac{N_{1}}{N}
$$

and

$$
E\left[D^{2}\right]=\frac{N_{2}}{N}
$$

we arrive to

$$
\kappa=\frac{E\left[D^{2}\right]}{E[D]}=\frac{N_{2}}{N_{1}}=\frac{N_{3}}{N_{2}} .
$$

The bound in (12) yields (15).

The spectral radius $\lambda_{1}$ can be approximated by its lower bound $N_{3} / N_{2}$, equivalently by $\kappa$ in an Erdös-Rényi random graph (if $p$ is high) or a Bárabasi-Albert graph, which proves the strong correlation between $\lambda_{1}$ and $\kappa$.

\subsection{Analytic relations in Erdös-Rényi random graphs}

Relation 6. In Erdös-Rényi random graphs $G_{p}(N)$, we have

$$
E[H] \approx \begin{cases}2-C_{G}, & \text { when } p \text { is large } \\ \frac{\log N}{\log N+\log C_{G}}, & \text { when } p \text { is small. }\end{cases}
$$


Proof. The average clustering coefficient of an Erdös-Rényi random graph $G_{p}(N)$ is

$$
E\left[C_{G}\right]=p .
$$

When the link density $p$ is large in Erdös-Rényi random graphs, the average hopcount [46] is

$$
E[H] \simeq 2-p .
$$

From (16) and (17), we obtain

$$
E[H] \simeq 2-C_{G}
$$

When $p$ is small, around the disconnectivity threshold $p_{c}$, we have [46]

$$
E[H] \approx \frac{\log N}{\log N+\log p} \approx \frac{\log N}{\log N+\log C_{G}} .
$$

Hence, relation 6 explains why the average hopcount $E[H]$ is negatively and strongly correlated with the clustering coefficient $C_{G}$ in Erdös-Rényi random graphs.

Relation 7. In Erdös-Rényi random graphs $G_{p}(N)$, we have

$$
\begin{array}{ll}
E\left[\lambda_{1}\right] \approx(2-E[H])(N-2), & \text { when } p \text { is large } \\
E[H] \approx \frac{\log N}{\log E\left[\lambda_{1}\right]}, & \text { when } p \text { is small. }
\end{array}
$$

Proof. The average of spectral radius $\lambda_{1}$ in Erdös-Rényi random graphs can be expressed as [49]

$$
E\left[\lambda_{1}\right]=p(N-2)+1+O\left(\frac{1}{\sqrt{N}}\right) .
$$

When $p$ is large, via (17), we have

$$
E\left[\lambda_{1}\right] \approx(2-E[H])(N-2)+1=C_{G}(N-2)+1 .
$$

When $p$ is around $p_{c}$, using (18)

$$
E[H] \approx \frac{\log N}{\log N+\log \left(E\left[\lambda_{1}\right] /(N-2)\right)}=\frac{\log N}{\log N-\log (N-2)+\log E\left[\lambda_{1}\right]} \approx \frac{\log N}{\log E\left[\lambda_{1}\right]} .
$$

Relation 7 supports the strong negative correlation between the spectral radius and the average hopcount. In dense Erdös-Rényi random graphs, (20) shows the strong positive correlation between the spectral radius and the clustering coefficient.

\subsection{Analytic relations in Bárabasi-Albert graphs}

Lemma 2. For Bárabasi-Albert graphs, it holds that

$$
\lambda_{1} \geq 2 C_{G}^{-1 / 3} \text {. }
$$


Proof. In the Bárabasi-Albert graph, when $m=4$, the clustering coefficient $C_{G}$ decreases [2] with the network size $N$ as

$$
C_{G} \approx N^{-3 / 4} \text {. }
$$

In addition, when the number of added links $m$ is equal to the starting number of vertices $m_{0}$, the maximum degree of the Bárabasi-Albert can be given as

$$
D_{\max }=m N^{1 / 2} \text {. }
$$

The lower bound of the spectral radius $\lambda_{1}$ can be given as the square root of the network's largest degree $D_{\max }$ (see [49, art 54, p 55]), as

$$
\lambda_{1} \geq \sqrt{D_{\max }}=\sqrt{m} N^{1 / 4} .
$$

Hence, we arrive at (21).

Inequality (21) supports that the linear correlation coefficient $\rho\left(\lambda_{1}, C_{G}\right) \approx 1$ in figure B.1.

Relation 8. In Bárabasi-Albert graphs

$$
E[H]<\frac{1}{C_{G}} .
$$

Proof. In Bárabasi-Albert graphs, the average hopcount approximates [2]

$$
E[H] \approx \frac{\ln N}{\ln \ln N}
$$

When $N>4$

$$
\frac{\ln N}{\ln \ln N}<N^{3 / 4}
$$

with (22), we obtain (25).

The inequality (25) supports the simulation results in the left bottom diagram of figure 1 and right bottom diagram of figure B.1 that the average hopcount $E[H]$ and the clustering coefficient $C_{G}$ are negatively correlated.

\section{Metric correlations in network models}

In this section, we compute the linear correlation coefficient $\rho(i, j)$ between any two metrics $i$ and $j$ defined in section 2 in a large number of network instances of Erdös-Rényi random graphs, Bárabasi-Albert graphs as well as Watts-Strogatz small-world graphs. The matrix $\rho$ is called the correlation matrix. The absolute value $0 \leq|\rho(i, j)| \leq 1$ characterizes the strength of the correlation between the corresponding metrics $i$ and $j$. If $|\rho(i, j)|$ is close to zero, the two metrics are almost uncorrelated whereas a $|\rho(i, j)|$ close to 1 implies a strong correlation. We do not explore further the sign of $\rho(i, j)$ which reflects whether the correlation is positive or negative, because it is the strength $|\rho(i, j)|$ indicates to which extent a metric can be predicted from the other. Furthermore, we investigate how metric correlations change with network parameters, specifically, the network size $N$ and the link density $p$ of Erdös-Rényi random graphs and the network size $N$ of Bárabasi-Albert graphs and Watts-Strogatz small-world graphs. 
The correlation of metrics in complex networks with applications in functional brain networks
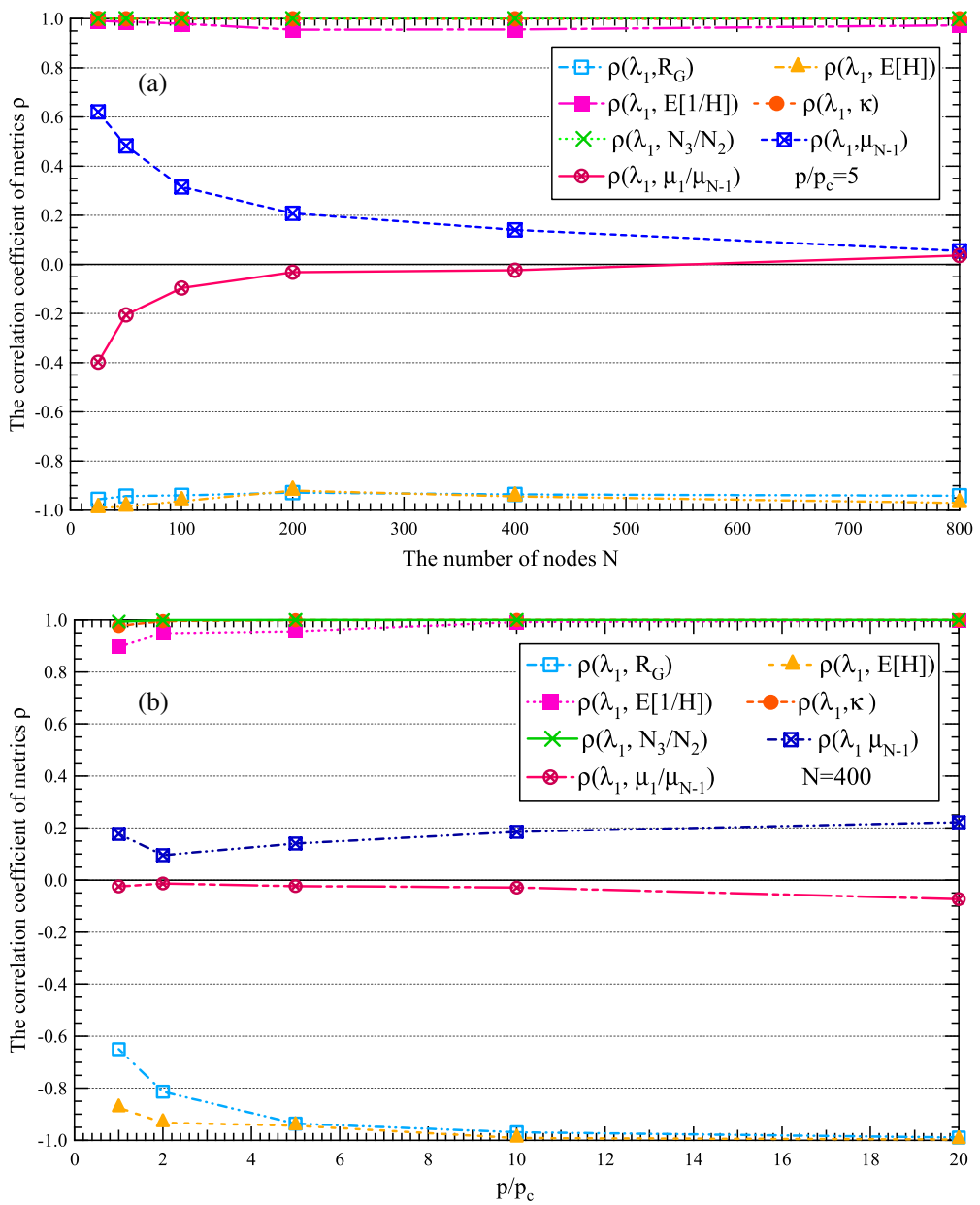

Figure 1. The correlation between the spectral radius and other metrics of ErdösRényi random graphs.

\subsection{Erdös-Rényi random graphs}

The Erdös-Rényi random graph $G_{p}(N)$ is a frequently occurring random complex network model, where $N$ is the number of nodes and $p$ is the link density between any two nodes. An Erdös-Rényi random graph is connected, if $p>p_{c} \approx \ln N / N$ for large $N$, where $p_{c}$ is the disconnectivity threshold. The correlation $\rho(i, j)$ between any two metrics is computed in the $10^{3}$ realizations of the Erdös-Rényi random graph $G_{p}(N)$, where $p=5 p_{c}$ and $N=25,50,100,200,400$ and 800. This allows us to explore how the metric correlation $\rho(i, j)$ evolves with the network size $N$. Similarly, the metric correlations are also computed in the $10^{3}$ instances of the Erdös-Rényi random graph $G_{p}(N)$, where $N=400$ and $p=\alpha p_{c}$ with $\alpha \in[1,2,5,10,20]$ to examine the influence of link density $p$ on metric correlations.

Figure 1 illustrates the linear correlation coefficient between $\lambda_{1}$ and other metrics for different size $N$ and link density $p$. The correlation between $\lambda_{1}$ and $\kappa, N_{3} / N_{2}$, $E[1 / H]$ are positive and strong over all network sizes and link densities: $\rho\left(\lambda_{1}, \kappa\right) \simeq$ $\rho\left(\lambda_{1}, N_{3} / N_{2}\right) \simeq \rho\left(\lambda_{1}, E[1 / H]\right) \simeq 1$. These three positive correlations are supported by analytic relations relation 5 , relation 1 , relation 7 and 3 , respectively. The correlation 
The correlation of metrics in complex networks with applications in functional brain networks

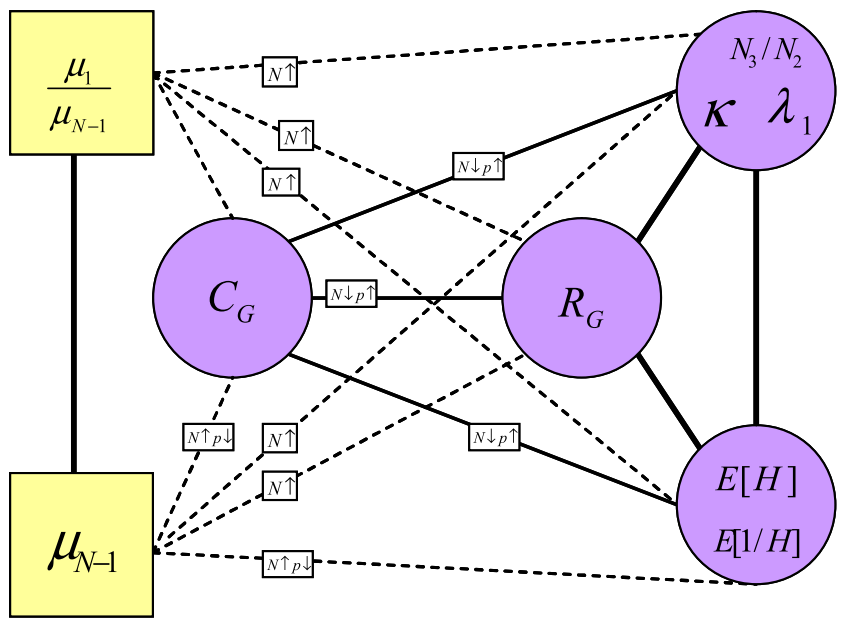

Figure 2. The metric correlation pattern in Erdös-Rényi random graphs..

between $\lambda_{1}$ and $R_{G}, E[H]$ are negative and strong $\rho\left(\lambda_{1}, R_{G}\right) \simeq \rho\left(\lambda_{1}, E[H]\right) \mid \simeq-1$ with the only condition that the link density should not be too small, thus $p \geq 4 p_{c}$. These observations can be analytically explained by lemma 1 and relation 7 , respectively. On the other hand, $\lambda_{1}$ tends to be independent or weakly correlated with $\mu_{N-1}$ and $\mu_{1} / \mu_{N-1}$ : $\left|\rho\left(\lambda_{1}, \mu_{1} / \mu_{N-1}\right)\right|<0.2$ and $\left|\rho\left(\lambda_{1}, \mu_{N-1}\right)\right|<0.2$, when the network size is large. Similarly, the correlation coefficient between any other two metrics as a function of the network size as well as the link density has been given in appendix A.

We consider two metrics $i$ and $j$ as uncorrelated if $|\rho(i, j)| \leq 0.2$ and as strongly correlated if $|\rho(i, j)| \geq 0.7$, because most of the correlations $|\rho(i, j)|$ are in the range $[0$, $0.2]$ and $[0.7,1]$. Since the correlation $\rho(i, j)$ changes with the network size $N$ and link density $p$, we claim two metrics are strongly correlated (or independent) if $|\rho(i, j)| \geq 0.7$ (or $|\rho(i, j)| \leq 0.2$ ) holds for a certain range of $N$ and $p$ and we will record the condition on $N$ and $p$, under which this strong (or weak) correlation is observed. In this way, we could obtain the metric correlation pattern based on results in figures 1, A.1 and A.2 and the correlation pattern is represented as a graph in figure 2 .

In correlation patterns, each node represents a metric. Two nodes are connected by a solid (or dotted) line if the corresponding metrics are strongly correlated (or independent) with $|\rho(i, j)| \geq 0.7$ (or $|\rho(i, j)| \leq 0.2$ ). The boxes along the links specify the conditions, if there are any, under which the strong or weak correlation has been observed. The up (down) arrow represents the value of $N$ or $p$ is large (or small). For example, when the size $N$ of the network is small and the link density $p$ is large, the correlation between $C_{G}$ and $R_{G}$ is strong. Metrics $\lambda_{1}, \kappa$ and $N_{3} / N_{2}$ are strongly mutually correlated and they have the same correlation coefficient with any other metric. Hence, we condense these three metrics into one node. The same holds for $E[H]$ and $E[1 / H]$.

Interestingly, our approach allows us to cluster the metrics into two, as marked by different shapes of nodes, when the size $N$ of the network is small and link density $p$ is large. Within each cluster, metrics are mutually strongly correlated (nodes are fully connected by solid lines). Moreover, any two metrics from different clusters are independent (any two nodes with different shapes are linked by a dotted line). Thus, it is sufficient to characterize an Erdös-Rényi random graph by two metrics, each from a different cluster, 
The correlation of metrics in complex networks with applications in functional brain networks

Table 1. Verification of the observed metric correlations in Erdös-Rényi random graph by analytic relations between metrics in section 3 .

\begin{tabular}{|c|c|c|c|c|c|c|c|c|c|}
\hline$\rho(i, j)$ & $R_{G}$ & $\mu_{N-1}$ & $\lambda_{1}$ & $\frac{\mu_{1}}{\mu_{N-1}}$ & $E[H]$ & $E\left[\frac{1}{H}\right]$ & $C_{G}$ & $\kappa$ & $N_{3} / N_{2}$ \\
\hline$R_{G}$ & 1 & & L1 & & $\mathrm{R} 4$ & R3, R4 & $\mathrm{R} 4, \mathrm{R} 6$ & L1, R5 & $\mathrm{L} 1, \mathrm{R} 1$ \\
\hline $\begin{array}{l}\mu_{N-1} \\
\lambda_{1} \\
\mu_{1} \\
\end{array}$ & L1 & 1 & 1 & $\mathrm{R} 2$ & $\mathrm{R} 7$ & $\mathrm{R} 3, \mathrm{R} 7$ & R6, R7 & $\mathrm{R} 5$ & $\mathrm{R} 1$ \\
\hline $\begin{array}{l}\mu_{N-1} \\
E[H]\end{array}$ & $\mathrm{R} 4$ & & $\mathrm{R} 7$ & & 1 & R3 & $\mathrm{R} 6$ & $\mathrm{R} 5, \mathrm{R} 7$ & $\mathrm{R} 1, \mathrm{R} 7$ \\
\hline$E\left[\frac{1}{H}\right]$ & $\mathrm{R} 3, \mathrm{R} 4$ & & R3, R7 & & R3 & 1 & $\mathrm{R} 3, \mathrm{R} 6$ & $\mathrm{R} 3, \mathrm{R} 5, \mathrm{R} 7$ & $\mathrm{R} 1, \mathrm{R} 3, \mathrm{R} 7$ \\
\hline $\mathcal{C}_{G}$ & $\mathrm{R} 4, \mathrm{R} 6$ & & $\mathrm{R} 6, \mathrm{R} 7$ & & $\mathrm{R} 6$ & $\mathrm{R} 3, \mathrm{R} 6$ & 1 & $\mathrm{R} 5, \mathrm{R} 6, \mathrm{R} 7$ & $\mathrm{R} 1, \mathrm{R} 6, \mathrm{R} 7$ \\
\hline$\kappa$ & $\mathrm{L} 1, \mathrm{R} 5$ & & $\mathrm{R} 5$ & & $\mathrm{R} 5, \mathrm{R} 7$ & $\mathrm{R} 3, \mathrm{R} 5, \mathrm{R} 7$ & $\mathrm{R} 5, \mathrm{R} 6, \mathrm{R} 7$ & 1 & $\mathrm{R} 1$ \\
\hline$N_{3} / N_{2}$ & $\mathrm{~L} 1, \mathrm{R} 1$ & & $\mathrm{R} 1$ & & $\mathrm{R} 1, \mathrm{R} 7$ & $\mathrm{R} 1, \mathrm{R} 3, \mathrm{R} 7$ & $\mathrm{R} 1, \mathrm{R} 6, \mathrm{R} 7$ & $\mathrm{R} 1$ & 1 \\
\hline
\end{tabular}

instead of by the nine metrics studied. In large and sparse networks, the correlation between clustering coefficient and the metrics in a circle is not strong any more. In that case, the representative set should contain three metrics: the clustering coefficient $C_{G}$, one metric in a rectangle $\left(\mu_{1} / \mu_{N-1}\right.$ or $\left.\mu_{N-1}\right)$ and one metric from the circle cluster $\left(R_{G}\right.$, $\lambda_{1}, \kappa, N_{3} / N_{2}, E[H]$ or $\left.E[1 / H]\right)$.

Those strong and weak metric correlations observed in Erdös-Rényi random graphs are supported by the analytic relations between metrics in section 3 as shown in table 1 .

\subsection{Bárabasi-Albert graphs}

The Bárabasi-Albert graph is one of the most studied network models since it generates a power-law degree distribution. Approximate power-law degree distributions are widely observed in complex networks. The mechanisms in Bárabasi-Albert graphs can also explain the origin of the social and economic disparities governing competitive systems [3]. The linear correlation coefficients between any two metrics are computed in $10^{3}$ instances of the BA model with each given set of parameters $m$ and $N$, where $m=4$ and $N=25,50,100,200,400$ and 800 . The metric correlations as a function of the network size $N$ are illustrated in figure B.1 in appendix B. Based on these results, we obtain the metric correlation pattern in Bárabasi-Albert graphs as depicted in figure 3.

We first consider the case when the network size $N$ is large. The metrics in a circle are mutually strongly correlated with each other and they are uncorrelated with the other metrics. Hence, one and only one metric in the circle cluster should be included in the representative set of metrics. On the other hand, the three metrics in a rectangle, $\mu_{1} / \mu_{N-1}, \mu_{N-1}$ and $R_{G}$ are not strongly correlated in large networks. Thus, these three metrics should all be included in the representative set. In summary, four metrics, the three marked as rectangle and one in circle, suffice to represent a power-law graph. When the network size is small, it is sufficient to characterize a network by three metrics $\kappa\left(\lambda_{1}\right.$ or $\left.N_{3} / N_{2}\right), E[H]$ (or $E[1 / H]$ ) and one metric from the rectangle cluster $\left(\mu_{1} / \mu_{N-1}, \mu_{N-1}\right.$ and $R_{G}$ ). This observed correlation pattern is supported by the analytic relations between metrics in section 3 as summarized in table 2 . 
The correlation of metrics in complex networks with applications in functional brain networks

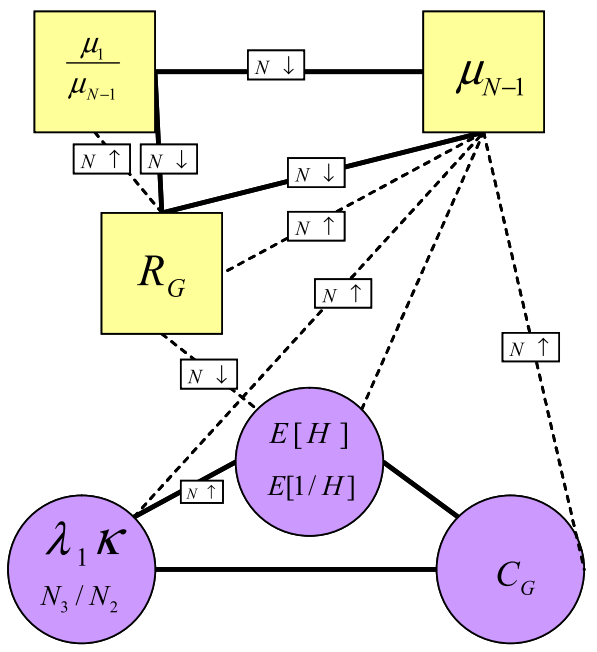

Figure 3. The metric correlation pattern in Bárabasi-Albert graphs..

\subsection{Watts-Strogatz small-world graphs}

A small-world graph refers to properties: (1) the average short path $E[H]$ is small, like that in an Erdös-Rényi random graph; and (2) the clustering coefficient $C_{G}$ is high, like that in a ring lattice where each node is adjacent to the $j$ following previous nodes. The structural properties of small-world networks have also been found in real-world networks, including social networks [32], neural networks [43], biological oscillators [24] and information propagation [30]. The small-world graphs of Watts and Strogatz [52] can be generated from a ring lattice with $N$ nodes and $k$ edges per node by rewiring each link at random with probability $p$. The linear correlation coefficients between any two metrics are computed in $10^{3}$ instances of the WS model with each given set of parameters $k, p$ and $N$, where $k=6, p=0.01$ and $N=200,400,800,1000$ and 2000. We choose the rewiring probability $p=0.01$ to generate graphs that have small-world properties, because: (1) in small-world rewiring, $C(p)$ is the clustering coefficient of the small-world graph with rewiring probability $p$ and $C(0)$ is the clustering coefficient of the ring lattice without rewiring. The dependence of the clustering coefficient ratio $C(p) / C(0)$ on $N$ is very small, and $C(p) / C(0)$ decreases with the fraction $p$, as $C(p) / C(0) \approx\left(1-p^{3}\right)[5]$; and (2) the average shortest path $E[H]$ is much smaller even when $p$ is small, as long as the size $N$ of the network is large enough [5]. The correlation coefficient between any other two metrics as a function of the network size has been given in figure C.1 in appendix C. With these results, we obtain the metric correlation pattern in Watts-Strogatz small-world graphs in figure 4 .

We first consider the case when the network size $N$ is small. In the circle cluster, metrics, i.e. $R_{G}, \kappa\left(N_{3} / N_{2}\right), E[H](E[1 / H]) C_{G}$ and $\mu_{N-1}$, are mutually strongly correlated with each other, except that $\mu_{1} / \mu_{N-1}$ is uncorrelated with $\kappa\left(N_{3} / N_{2}\right)$ and $C_{G}$. Furthermore, $\lambda_{1}$ is only correlated with $\kappa\left(N_{3} / N_{2}\right)$ and the assortativity $\rho_{D}$ in a triangle is not correlated all other metrics in networks. Hence, four metrics should be included in the representative set: $\rho_{D}, \lambda_{1}, \mu_{1} / \mu_{N-1}$ and another metric in the circle cluster. When the network size is large, it is still enough to characterize a network by four metrics $\lambda_{1}$, $\mu_{1} / \mu_{N-1}\left(\right.$ or $\left.\mu_{N-1}\right)$, one from other metrics in a circle and $\rho_{D}$. 
Table 2. Verification of the observed metric relations in Bárabasi-Albert graph by analytic relations between metrics in section 3 .

\begin{tabular}{|c|c|c|c|c|c|c|c|c|c|}
\hline$\rho(i, j)$ & $R_{G}$ & $\mu_{N-1}$ & $\lambda_{1}$ & $\frac{\mu_{1}}{\mu_{N-1}}$ & $E[H]$ & $E\left[\frac{1}{H}\right]$ & $C_{G}$ & $\kappa$ & $N_{3} / N_{2}$ \\
\hline$R_{G}$ & 1 & & L1 & & $\mathrm{R} 4$ & $\mathrm{R} 3, \mathrm{R} 4$ & L1 and L2 & L1, R5 & $\mathrm{L} 1, \mathrm{R} 1$ \\
\hline $\begin{array}{l}\mu_{N-1} \\
\lambda_{1}\end{array}$ & L1 & 1 & 1 & $\mathrm{R} 2$ & L1 and R4 & $\mathrm{L} 1, \mathrm{R} 3, \mathrm{R} 4$ & L2 & $\mathrm{R} 5$ & $\mathrm{R} 1$ \\
\hline$\underline{\mu_{1}}$ & & $\mathrm{R} 2$ & & 1 & & & & & \\
\hline $\begin{array}{l}\mu_{N-1} \\
E[H]\end{array}$ & $\mathrm{R} 4$ & & $\mathrm{~L} 1, \mathrm{R} 4$ & & 1 & $\mathrm{R} 3$ & $\mathrm{R} 8$ & L1, L4, L5 & $\mathrm{L} 1, \mathrm{R} 1, \mathrm{R} 4$ \\
\hline$E \frac{1}{H}$ & $\mathrm{R} 3, \mathrm{R} 4$ & & $\mathrm{~L} 1, \mathrm{R} 3, \mathrm{R} 4$ & & $\mathrm{R} 3$ & 1 & $\mathrm{R} 3, \mathrm{R} 8$ & $\mathrm{~L} 1, \mathrm{R} 3, \mathrm{R} 4, \mathrm{R} 5$ & $\mathrm{~L} 1, \mathrm{R} 1, \mathrm{R} 3, \mathrm{R} 4$ \\
\hline $\mathcal{C}_{G}$ & $\mathrm{~L} 1, \mathrm{R} 2$ & & L2 & & $\mathrm{R} 8$ & R3 and R8 & 1 & $\mathrm{~L} 2, \mathrm{R} 5$ & $\mathrm{~L} 2, \mathrm{R} 1$ \\
\hline$\kappa$ & $\mathrm{L} 1, \mathrm{R} 5$ & & $\mathrm{R} 5$ & & $\mathrm{~L} 1, \mathrm{R} 4, \mathrm{R} 5$ & $\mathrm{~L} 1, \mathrm{R} 3, \mathrm{R} 4, \mathrm{R} 5$ & $\mathrm{~L} 2, \mathrm{R} 5$ & 1 & $\mathrm{R} 1$ \\
\hline$N_{3} / N_{2}$ & $\mathrm{~L} 1, \mathrm{R} 1$ & & $\mathrm{R} 1$ & & $\mathrm{~L} 1, \mathrm{R} 1, \mathrm{R} 4$ & $\mathrm{~L} 1, \mathrm{R} 1, \mathrm{R} 3, \mathrm{R} 4$ & $\mathrm{~L} 2, \mathrm{R} 1$ & $\mathrm{R} 1$ & 1 \\
\hline
\end{tabular}


The correlation of metrics in complex networks with applications in functional brain networks

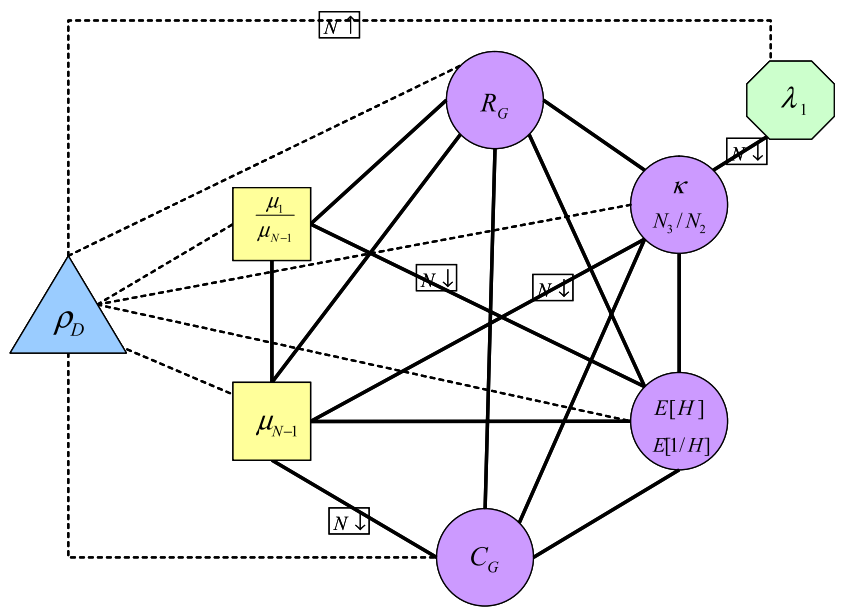

Figure 4. The metric correlation pattern in Watts-Strogatz small-world graphs..

The different metric correlation patterns observed in Erdös-Rényi random graphs (figure 2), Bárabasi-Albert graphs (figure 3) and Watts-Strogatz small-world graphs (figure 4) reflect that metric correlations depend on the graph, via the adjacency matrix, e.g. the degree distribution. Both network models can be characterized by a small set of three or four metrics instead of by the nine metrics studied. Surprisingly, the average hopcount $E[H]$ and clustering coefficient $C_{G}$, the most studied metrics so far especially in neuroscience turn out to be strongly correlated ${ }^{9}$. On the other hand, spectral metrics, which are investigated only recently, seem to be essential in network characterizations. At least, spectral metrics always appear in the representative set in these three network models.

\section{Metric correlations in functional brain networks}

It has become clear that properties (reflected by metrics) of brain networks may predict brain functioning such as cognitive performance [36, 39, 40,44]. Moreover, brain networks show systematic changes during development under genetic control and reveal characteristic patterns of disruption in various types of neurological disease. In order to understand which network property/metric is the most relevant to a certain brain functioning, it is essential to understand the relations between network properties. In this section, we investigate the metric correlation pattern in the functional brain networks of 22 healthy subjects. The correlation pattern observed in functional brain networks will be compared with what we discovered in network models.

The concept of functional connectivity refers to the statistical interdependences between physiological time series recorded in various brain areas, and is thought to reflect communication between several brain areas [1]. Magnetoencephalography (MEG), a recording of the brain's magnetic activity, is a method used to assess functional connectivity within the brain. A functional brain network is created by regarding each MEG channel as a node, and the functional connectivity between

\footnotetext{
${ }^{9}$ Note that this strong correlation is expected in a class of networks with the same degree distribution as in the three models, but not in graphs with different degree distributions.
} 
each pair of channels represents a link whose weight reflects the strength of the connectivity or correlation. Correlations between the time series of the channels were analyzed with the synchronization likelihood (SL), a nonlinear measure of statistical interdependences $[29,42]$. The functional brain networks were measured in 22 healthy people, whose mean age was 63.6, by the medical ethics committee of the VU Medical Center, Amsterdam. More information about the data can be found in [14]. Brain activity was measured by MEG with $N=149$ recording channels. With MEG, a loss of upper alpha-, beta-and gamma-band synchronization could be demonstrated in patients with Alzheimer's disease, both during an eye-closed state as well as during an eye-open state. In these studies, the $13-30 \mathrm{~Hz}$ beta-band showed the most consistent abnormalities in the subjects [43]. Hence, we focus on the 13-30 Hz band. The functional brain network was measured four times for each person in 13-30 Hz (beta) band. In total, we have 88 functional brain networks. Each network is a weighted complete graph, where each link weight $0 \leq w_{i j} \leq 1$.

Since we focus on metrics correlations in unweighted networks, each weighted network is transformed to an unweighted network mapped as the union of links whose link weight is above a threshold $T$. There are many methods to choose the threshold $T$ [50]. In this paper, we use either a fixed threshold $T$ for all networks or a network-dependent $T$ which makes the link density $p$ of all unweighted networks similar. The metric correlation patterns are studied in the two resulting classes of networks with different degree distributions.

\subsection{Metric correlations in unweighted functional brain networks transformed with fixed threshold $T$}

Stam et al [43] investigated the clustering coefficients and the shortest path lengths as a function of the threshold $T$ on functional brain networks, and gave some suggestions on how to set the threshold $T$. When the threshold $T$ is small, the corresponding unweighted functional brain networks are almost fully connected. If $T$ is large, the unweighted network tends to be disconnected. Regarding the studied functional brain networks, we find that, when $T \in[0.001,0.019]$, the corresponding unweighted functional brain networks are connected. When $T \geq 0.02$, not all the networks are connected. In summary, to avoid disconnected and fully connected graphs, we choose $T=0.019$ to transform each weighted functional brain network into an unweighted one, where we calculate the correlation between those metrics mentioned above. As shown in figure 5, the degree distribution of the unweighted functional brain networks is close to a binomial distribution, as in Erdös-Rényi random graphs.

The linear correlation coefficients between any two metrics in the unweighted functional brain networks are shown in table 3. The corresponding correlation pattern is depicted in figure 6. Since all the functional brain networks have the same size $N$, no condition is associated with any correlation or dependency. Instead, we place the correlation coefficient along each pair of metrics.

Both the functional brain networks and Erdös-Rényi random graphs possess a binomial degree distribution. Interestingly, the metric correlation patterns observed in both types of graphs as given in figures 2 and 6 are consistent with each other: (a) the same set of metrics in the circle cluster as well as in the rectangle cluster; and (b) the 
The correlation of metrics in complex networks with applications in functional brain networks

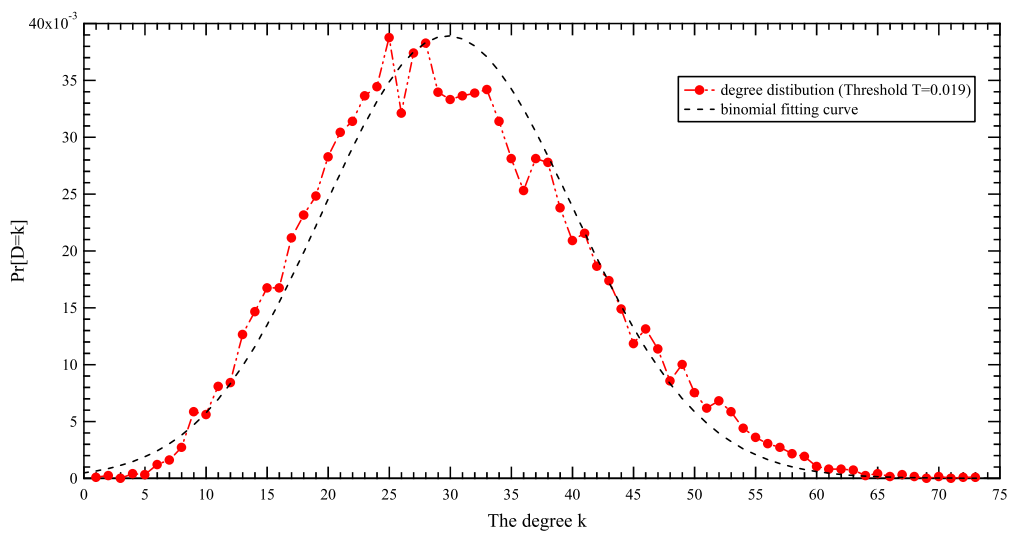

Figure 5. The degree distribution of unweighted functional brain networks (fixed $T=0.019)$ as well as its binomial curve fitting.

Table 3. The correlation coefficients between metrics in unweighted functional brain networks (healthy people, beta-band, fixed threshold $T=0.019$ ).

\begin{tabular}{lrrrrrrrrrrr}
\hline$\rho(i, j)$ & \multicolumn{1}{c}{$R_{G}$} & $\mu_{N-1}$ & $\lambda_{1}$ & $\frac{\mu_{1}}{\mu_{N-1}}$ & $E[H]$ & $E\left[\frac{1}{H}\right]$ & $C_{G}$ & $\rho_{D}$ & $\kappa$ & $N_{3} / N_{2}$ \\
\hline$R_{G}$ & 1.00 & -0.77 & -0.94 & 0.31 & 0.98 & -0.97 & -0.91 & -0.33 & -0.94 & -0.94 \\
$\mu_{N-1}$ & -0.77 & 1.00 & 0.66 & -0.73 & -0.73 & 0.72 & 0.60 & 0.23 & 0.67 & 0.67 \\
$\lambda_{1}$ & -0.94 & 0.66 & 1.00 & -0.11 & -0.97 & 0.98 & 0.92 & 0.37 & 1.00 & 1.00 \\
$\frac{\mu_{1}}{\mu_{N-1}}$ & 0.31 & -0.73 & -0.11 & 1.00 & 0.20 & -0.19 & -0.16 & -0.16 & -0.12 & -0.11 \\
$E[H]$ & 0.98 & -0.73 & -0.97 & 0.20 & 1.00 & -1.00 & -0.88 & -0.26 & -0.97 & -0.97 \\
$E\left[\frac{1}{H}\right]$ & -0.97 & 0.72 & 0.98 & -0.19 & -1.00 & 1.00 & 0.90 & 0.27 & 0.99 & 0.99 \\
$C_{G}$ & -0.91 & 0.60 & 0.92 & -0.16 & -0.88 & 0.90 & 1.00 & 0.46 & 0.91 & 0.92 \\
$\rho_{D}$ & -0.33 & 0.23 & 0.37 & -0.16 & -0.26 & 0.27 & 0.46 & 1.00 & 0.31 & 0.34 \\
$\kappa$ & -0.94 & 0.67 & 1.00 & -0.11 & -0.97 & 0.99 & 0.91 & 0.31 & 1.00 & 1.00 \\
$N_{3} / N_{2}$ & -0.94 & 0.67 & 1.00 & -0.11 & -0.97 & 0.99 & 0.92 & 0.34 & 1.00 & 1.00 \\
\hline
\end{tabular}

size of functional brain networks $N=149$ is small. In small Erdös-Rényi random graphs, the clustering coefficient is strongly correlated with the other metric in a circle, moreover $\rho\left(\mu_{N-1}, R_{G}\right)$ and $\rho\left(\mu_{N-1}, E[H]\right)$ are not small, the same as observed in the functional brain networks. The only difference is that the assortativity $\rho_{D}$ is usually considered in real-world complex networks but not in network models where $\rho_{D} \rightarrow 0$. Actually, functional brain networks are assortative $\rho_{D}>0$. The representative set $\rho_{D}, \mu_{1} / \mu_{N-1}$ and $R_{G}$ is preferable due to the independence between $\mu_{1} / \mu_{N-1}$ and metrics in a circle as well as the strong correlation between $R_{G}$ and many other metrics. The consistency in metric correlation patterns (figures 2 and 6) in networks with a same degree distribution verifies the crucial influence of the network property, especially the degree distribution, on the relations between metrics. Importantly, both the degree distribution and the metric correlation pattern suggest this particular class of MEG unweighted functional brain networks with a fixed threshold are almost Erdös-Rényi graph-like, and not smallworld. 
The correlation of metrics in complex networks with applications in functional brain networks

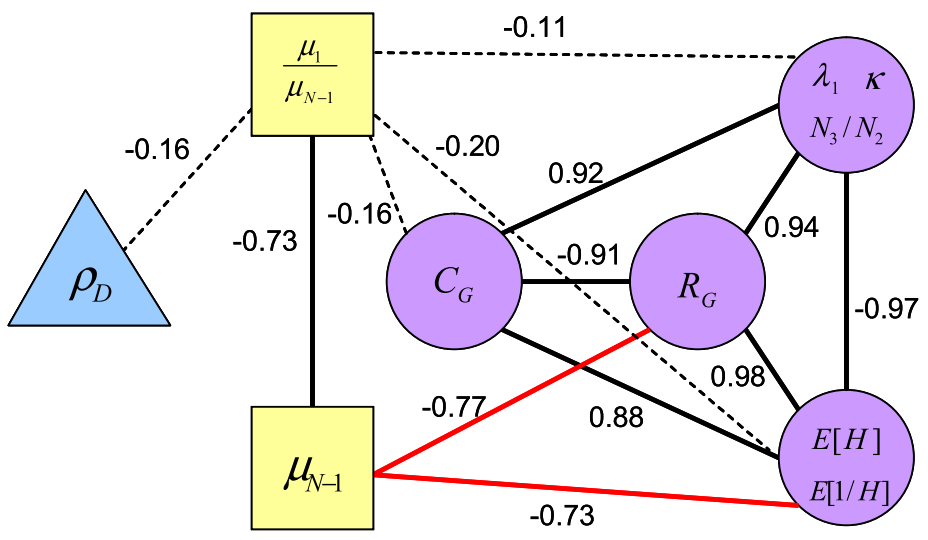

Figure 6. The metric correlation pattern of unweighted functional brain networks (fixed threshold $T$ ).

\subsection{Metric correlations in unweighted functional brain networks transformed with fixed link density $p$}

Van Wijk et al [50] claimed that graph measures can be influenced by the number $N$ of nodes and the link density $p$. It is easier to compare networks, such as the functional brain networks, with the same size $N$ and link density $p$. Thus, we choose a threshold $T$ for each weighted network such that the average degree of the corresponding unweighted network equals a given constant value. In this paper, we choose the threshold such that the average degree equals 15. Each unweighted network follows almost the same degree distribution. The degree distribution of the unweighted functional brain networks is shown in figure 7, which has a heavy tail. It differs from the degree distribution of any of the three network models mentioned in section 4 . The linear correlation coefficients between any two metrics in the unweighted functional brain networks are shown in table 4 . The corresponding correlation pattern is depicted in figure 8 .

The metrics can be divided into three groups, which are shaped rectangle, circle and triangle. Metrics in the same shape are strongly correlated with each other, while metrics in different shapes are not strongly correlated. We could choose one metric from each shape group, therefore three representative metrics can characterize the unweighted functional brain networks.

\section{Conclusion}

In this paper we have studied the correlations between widely studied metrics in functional brain networks as well as in three classical complex network models, namely Erdös-Rényi, Bárabasi-Albert graphs and Watts-Strogatz small-world graphs. The metric correlation pattern in each of the three classes of graphs illustrates the strong correlations and independences between metrics, which indicates the possibility to determine a small set of representative metrics by including only one metric from each subset of mutually strongly dependent metrics. This representative set of metrics tends to characterize both sufficiently and effectively a class of networks with a given degree distribution. When we study a specific network, however, the representative set at least has to be 
The correlation of metrics in complex networks with applications in functional brain networks
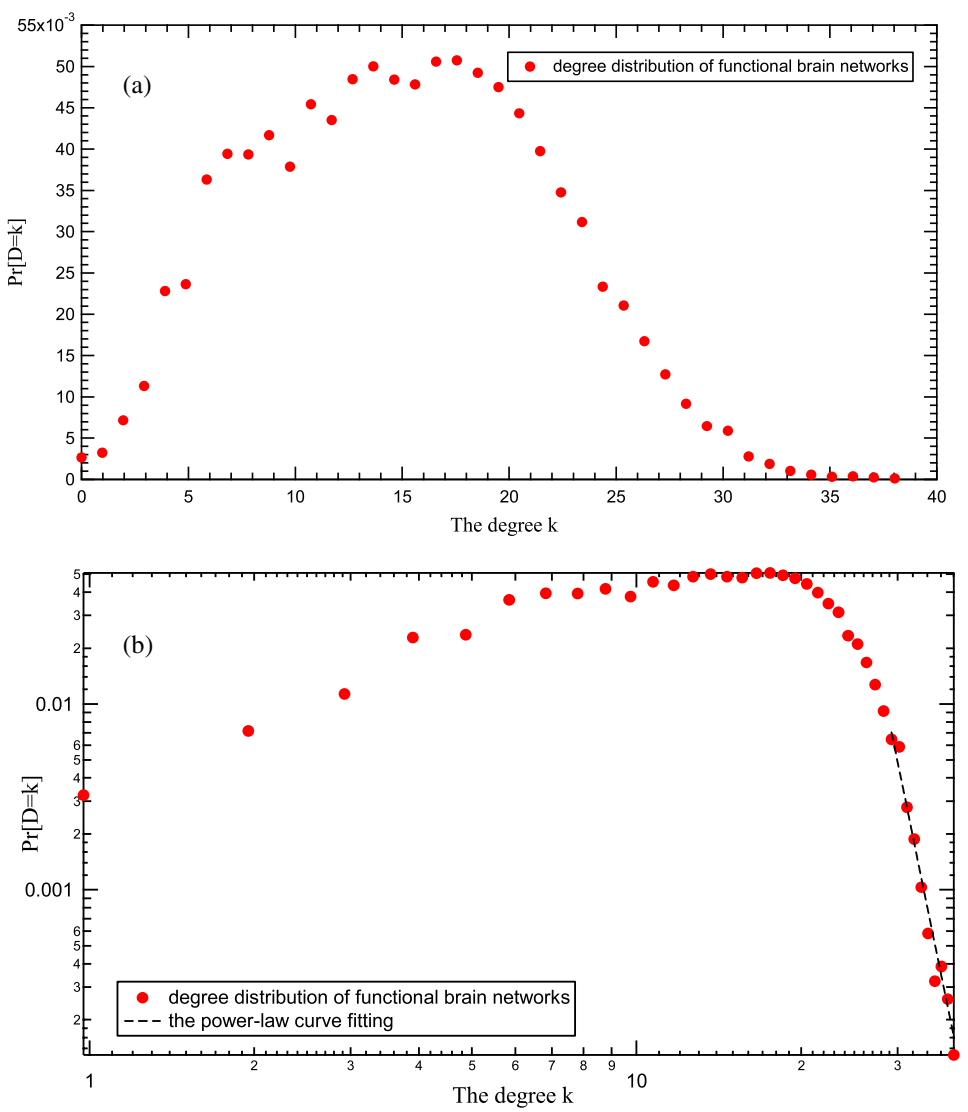

Figure 7. The degree distribution of unweighted functional brain networks (fixed link density $p$ ).

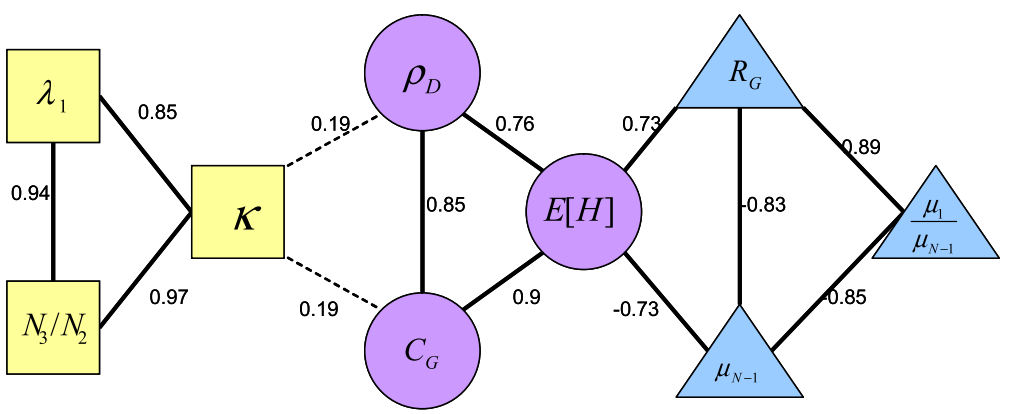

Figure 8. The metric correlation pattern of unweighted functional brain networks (fixed link density $p$ ).

considered so that important network properties will not be overlooked. Most of the metric correlations observed so far are supported/explained analytically by theorems developed in this paper as well as in the literature. Furthermore, graphs with a similar degree distribution, such as the unweighted functional brain networks with fixed threshold $T$ and Erdös-Rényi random graphs, tend to possess a similar metric correlation pattern, which verifies the influence of the degree distribution on metric relations. When the degree distribution of networks is different, the metric patterns are also different. Hence, 
The correlation of metrics in complex networks with applications in functional brain networks

Table 4. The correlation coefficients between metrics in unweighted functional brain networks (healthy people, beta-band, same size $(L$ and $N)$ ).

\begin{tabular}{lrrrrrrrrrr}
\hline$C_{i j}$ & $R_{G}$ & $\mu_{N-1}$ & $\lambda_{1}$ & $\frac{\mu_{1}}{\mu_{N-1}}$ & $E[H]$ & $E\left[\frac{1}{H}\right]$ & $C_{G}$ & $\rho_{D}$ & $\kappa$ & $N_{3} / N_{2}$ \\
\hline$R_{G}$ & 1.00 & -0.83 & 0.61 & 0.89 & 0.73 & -0.69 & 0.54 & 0.47 & 0.64 & 0.68 \\
$\mu_{N-1}$ & -0.83 & 1.00 & -0.51 & -0.85 & -0.73 & 0.72 & -0.63 & -0.51 & -0.47 & -0.52 \\
$\lambda_{1}$ & 0.61 & -0.51 & 1.00 & 0.56 & 0.48 & -0.47 & 0.44 & 0.55 & 0.85 & 0.94 \\
$\frac{\mu_{1}}{\mu_{N-1}}$ & 0.89 & -0.85 & 0.56 & 1.00 & 0.67 & -0.61 & 0.46 & 0.41 & 0.55 & 0.59 \\
$E[H]$ & 0.73 & -0.73 & 0.48 & 0.67 & 1.00 & -0.99 & 0.90 & 0.76 & 0.30 & 0.42 \\
$E\left[\frac{1}{H}\right]$ & -0.69 & 0.72 & -0.47 & -0.61 & -0.99 & 1.00 & -0.94 & -0.81 & -0.25 & -0.39 \\
$\mathcal{C}_{G}$ & 0.54 & -0.63 & 0.44 & 0.46 & 0.90 & -0.94 & 1.00 & 0.85 & 0.19 & 0.34 \\
$\rho_{D}$ & 0.47 & -0.51 & 0.55 & 0.41 & 0.76 & -0.81 & 0.85 & 1.00 & 0.19 & 0.39 \\
$\kappa$ & 0.64 & -0.47 & 0.85 & 0.55 & 0.30 & -0.25 & 0.19 & 0.19 & 1.00 & 0.97 \\
$N_{3} / N_{2}$ & 0.68 & -0.52 & 0.94 & 0.59 & 0.42 & -0.39 & 0.34 & 0.39 & 0.97 & 1.00 \\
\hline
\end{tabular}

a specific representative set of metrics should be determined for a particular degree distribution. Luckily, degree distribution is the simplest metric to compute. Finally, two observations are considered important especially for the applications of network science to other disciplines: (i) the average distance and the clustering coefficient, the most studied metrics so far in neuroscience, are strongly correlated and, thus, redundant; and (ii) spectral metrics, though only studied recently in the context of complex networks, seem to be essential in network characterizations.

Note that the metric correlations are studied and observed in networks with a similar degree distribution. These results cannot simply be applied to a class of networks with different degree distributions. The representative set tends to be larger when more than nine metrics are considered. So far, we have explored the effect of degree distribution on metric correlations. It is interesting to further examine a large set of metrics and the metric correlation pattern in graphs with a given degree distribution and a given degree correlation (assortativity).

Topologies of many complex networks such as the Internet at router level are not known due to their large size or technical challenges, although some properties of their subnetworks (i.e. within different autonomous systems) can be measured. The dependence of metric correlation pattern on network properties opens up a new direction of reverse engineering: we infer possible universal network properties or the possible suitable network model of a class of graphs from the correlations of the measured network metrics.

\section{Acknowledgments}

This research was supported by Next Generation Infrastructures (Bsik), the EU FP7 project ResumeNet (project no. 224619) and the China Scholarship Council (CSC).

\section{Appendix A. Metric correlations in Erdös-Rényi random graphs (figures A.1 and A.2)}


The correlation of metrics in complex networks with applications in functional brain networks
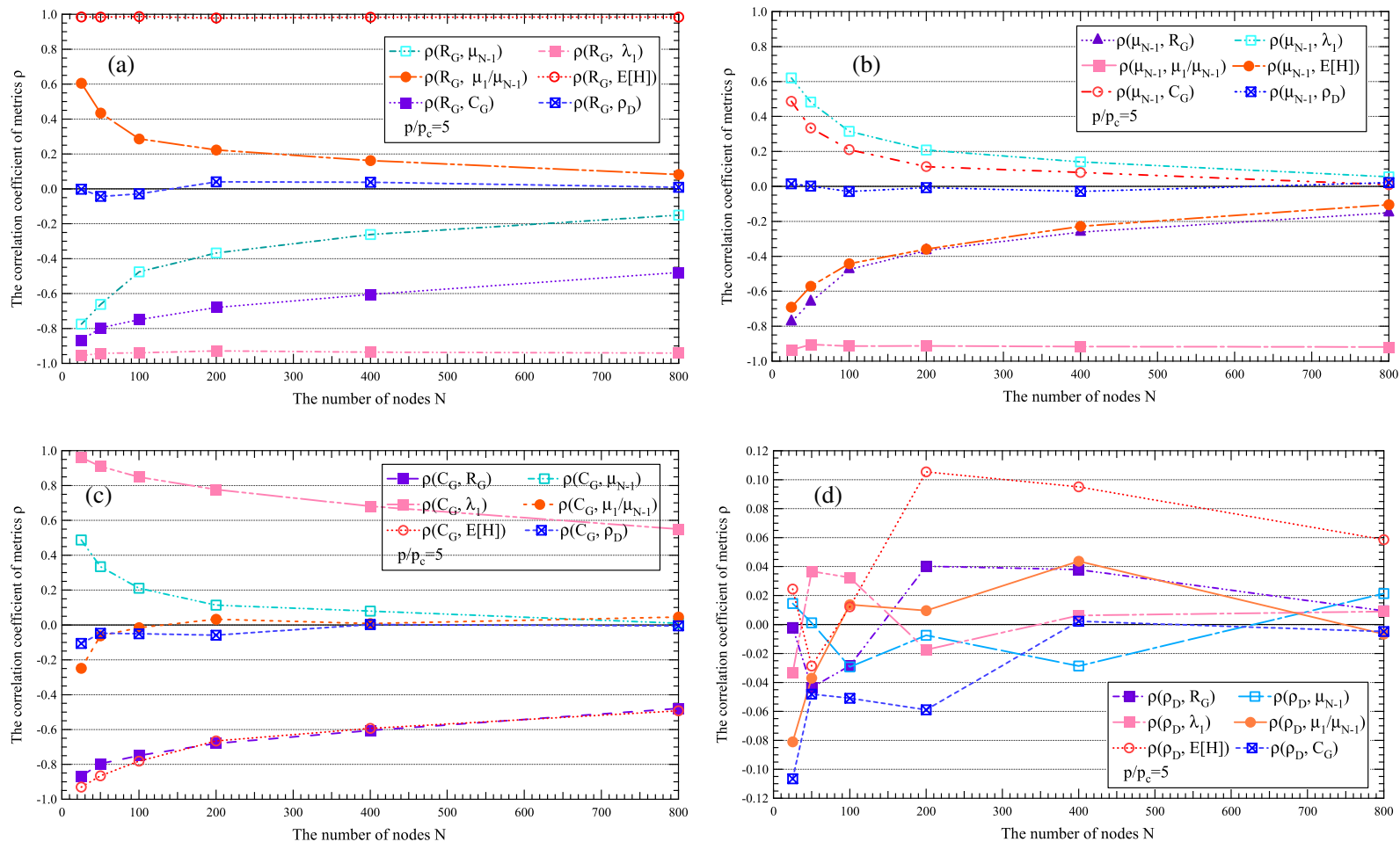

Figure A.1. Metric correlations as a function of $N$ in Erdös-Rényi random graphs $(p=5 \mathrm{pc})$.
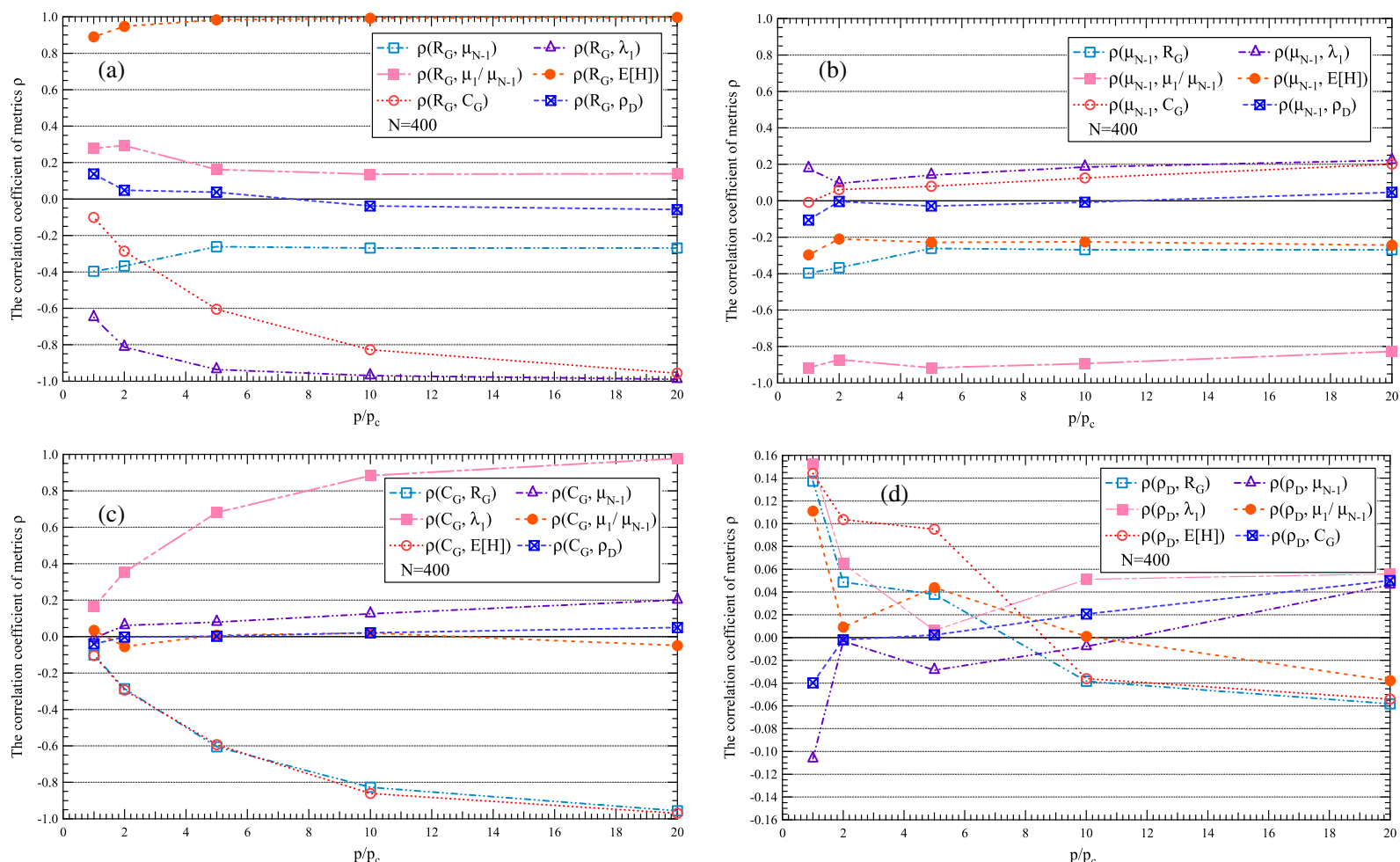

Figure A.2. Metric correlations as a function of $p$ in Erdös-Rényi random graphs $(N=400)$. 
The correlation of metrics in complex networks with applications in functional brain networks

\section{Appendix B. Metric correlations in Bárabasi-Albert graphs (figure B.1)}
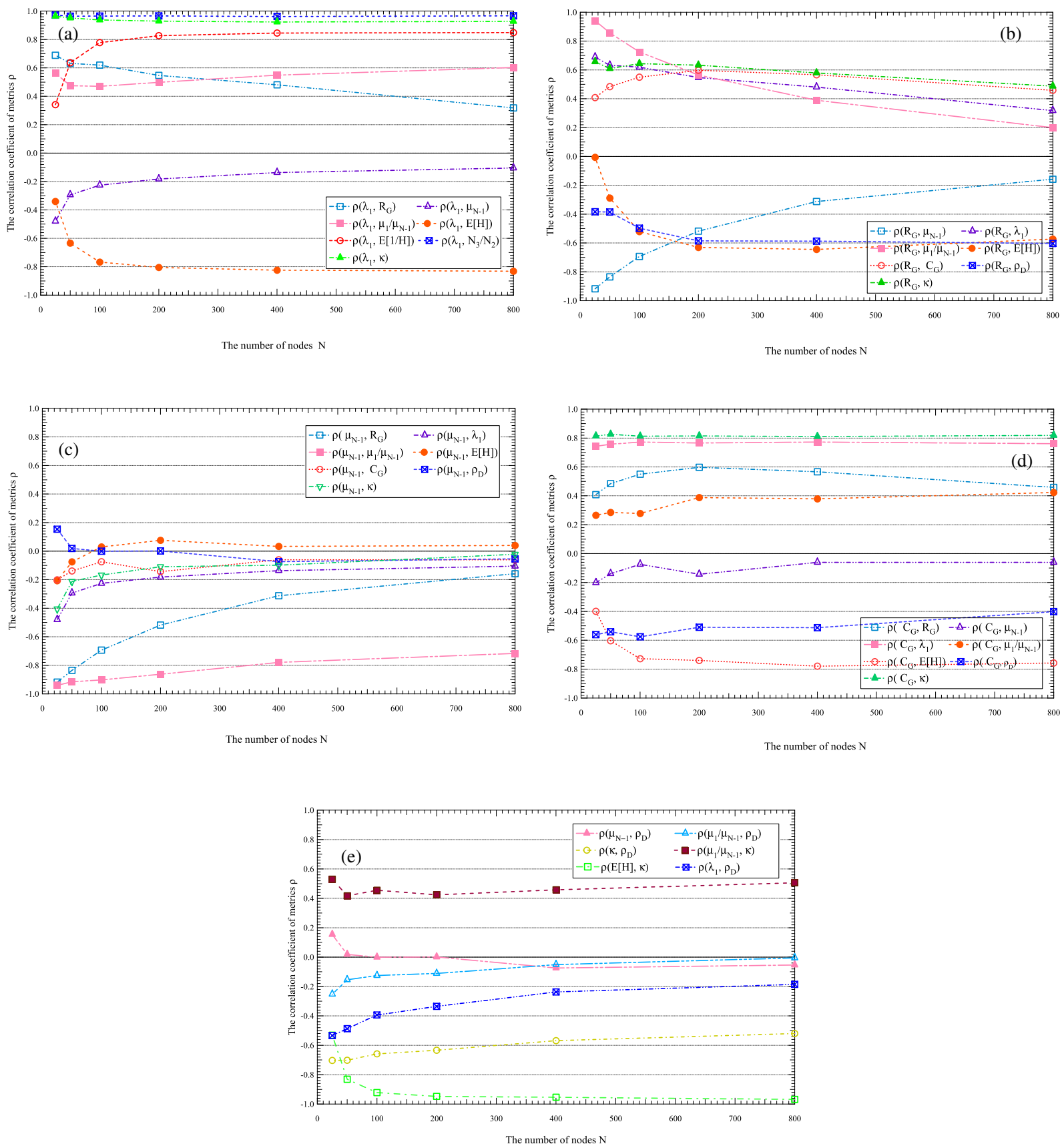

Figure B.1. Metric correlations as a function of $N$ in Bárabasi-Albert graphs $(m=4)$. 
The correlation of metrics in complex networks with applications in functional brain networks

\section{Appendix C. Metric correlations in Watts-Strogatz small-world graphs (figure C.1)}
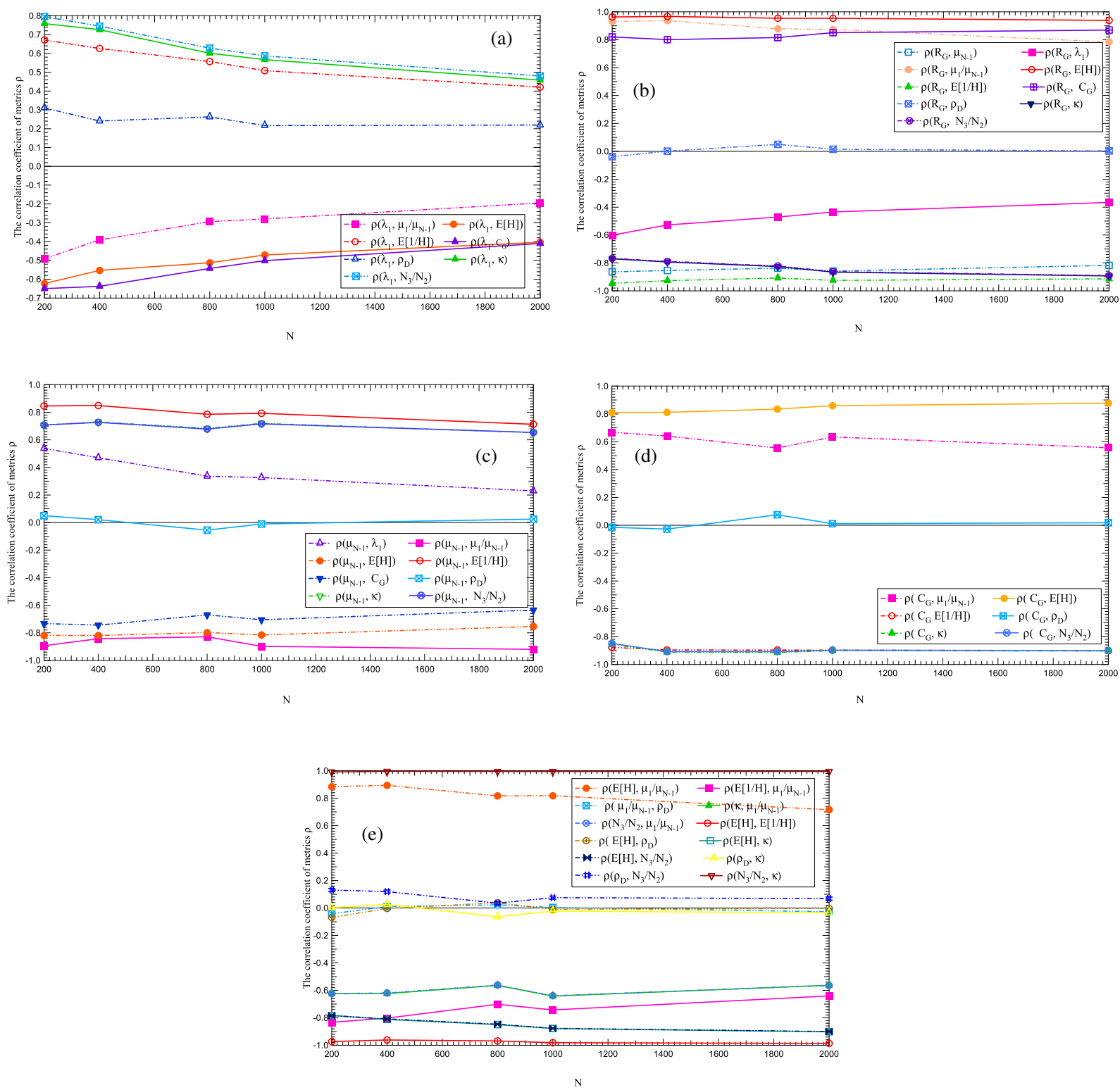

Figure C.1. Metric correlations as a function of $N$ in Watts-Strogatz small-world graphs $(k=6, p=0.01)$.

\section{References}

[1] Aertsen A M, Gerstein G L, Habib M K and Palm G, Dynamics of neuronal firing correlation: modulation of 'effective connectivity', 1989 J. Neurophysiol. 61900

[2] Albert R and Bárabasi A-L, Statistical mechanics of complex networks, 2002 Rev. Mod. Phys. 7447

[3] Barabasi A-L and Albert R, Emergency of scaling in random networks, 1999 Science 286509

[4] Barahona M and Pecora L M, Synchronization in small-world systems, 2002 Phys. Rev. Lett. 89054101

[5] Barrat A and Weigt M, On the properties of small-world network models, 2000 Eur. Phys. J. B 13546 
The correlation of metrics in complex networks with applications in functional brain networks

[6] Barrat A, Barthelemy M and Vespignani A, 2008 Dynamical Processes on the Complex Networks (Cambridge: Cambridge University Press)

[7] Bartolomei F et al, Disturbed functional connectivity in brain tumour patients: evaluation by graph analysis of synchronization matrices, 2006 Clin. Neurophysiol. 1172039

[8] Bullmore E and Sporns O, Complex brain networks: graph theoretical analysis of structural and functional systems, 2009 Nature Rev. Neurosci. 10186

[9] Chavez M, Valencia M, Navarro V, Latora V and Martinerie J, Functional modularity of background activities in normal and epileptic brain networks, 2010 Phys. Rev. Lett. 104118701

[10] Chung F, Lu L and Vu V, The spectra of random graphs with given expected degrees, 2003 Proc. Nat. Acad. Sci. 1006313

[11] Cohen R and Havlin S, 2010 Complex Networks-Structure, Robustness and Function (Cambridge: Cambridge University Press)

[12] Costa L da F, Rodrigues F A, Travieso G and Villas Boas P R, Characterization of complex networks: a survey of measurements, 2007 Adv. Phys. 56167

[13] de Haan W, Pijnenburg Y A, Strijers R L, van der Made Y, van der Flier W M, Scheltens P and Stam C J, Functional neural network analysis in frontotemporal dementia and Alzheimer's disease using EEG and graph theory, 2009 BMC Neurosci. 10101

[14] de Haan W, Van der Flier W M, Scheltens P and Stam C J, Resting-state functional modularity is disturbed in Alzheimer's disease, 2011 NeuroImage at press

[15] de la Fuente A, Bing N, Hoeschele I and Mendes P, Discovery of meaningful associations in genomic data using partial correlation coefficients, 2004 Bioinformatics 203575

[16] Donetti L, Neri F and Muñoz M A, Optimal network topologies: expanders, cages, ramanujan graphs, entangled networks and all that, 2006 J. Stat. Mech. P08007

[17] Ellens W, Spieksma F M, Van Mieghem P, Jamakovic A and Kooij R E, Effective graph resistance, 2011 Linear Algebr. Appl. 4352491

[18] Erdös P and Rényi A, On random graphs, I, 1959 Publ. Math. Debrecen 6290

[19] Fiedler M, Algebraic connectivity of graphs, 1973 Czech. Math. J. 23298

[20] Ghosh A, Boyd S and Saberi A, Minimizing effective resistance of a graph, 2008 SIAM Rev. 5037

[21] Jamakovic A, Uhlig S and Theisler I, On the relationship between topological metrics in real-world networks, 2007 Proc. 4th Eur. Conf. on Complex Systems (ECCS 07, Dresden)

[22] Jamakovic A and Van Mieghem P, On the robustness of complex networks by using the algebraic connectivity, 2008 Proc. IFIP Networking Conf. (Singapore City)

[23] Klein D J and Randic M, Resistance distance, 1993 J. Math. Chem. 1281

[24] Kuramoto Y, 1984 Chemical Oscillations, Waves and Turbulence (Berlin: Springer)

[25] Latora V and Marchiori M, Efficient behavior of small-world networks, 2001 Phys. Rev. Lett. 8719

[26] Li Y, Liu Y, Li J, Qin W, Li K, Yu Ch and Jiang T, Brain anatomical network and intelligence, 2009 PloS Comput. Biol. 5 e1000395

[27] Lynall M-E, Bassett D S, Kerwin R, McKenna P J, Kitzbichler M, Muller U and Bullmore E, Functional connectivity and brain networks in schizophrenia, 2010 J. Neurosci. 309477

[28] Mohar B, Alavi Y, Chartrand G, Oellermann O R and Chwenk A J, The laplacian spectrum of graphs, 1991 Graph Theory, Comb. Appl. 2871

[29] Montez T, Linkenkaer-Hansen K, van Dijk B W and Stam C J, Synchronization likelihood with explicit time-frequency priors, 2006 Neuroimage 331117

[30] Moore C and Newman M E J, Exact solution of site and bond percolation on small-world networks, 2000 Phys. Rev. E 627059

[31] Moreno Y, Pastor-Satorras R and Vespignani A, Epidemic outbreaks in complex heterogeneous networks, 2002 Eur. Phys. J. B 26521

[32] Newman M E J and Watts D J, Renormalization group analysis of the small-world network model, 1999 Phys. Lett. A 263341

[33] Newman M E J, Assortative mixing in networks, 2002 Phys. Rev. Lett. 89208701

[34] Newman M E J, Mixing patterns in networks, 2003 Phys. Rev. E 67026126

[35] Nishikawa T, Motter A E, Lai Y-C h and Hoppensteadt F C, Heterogneity in oscillator networks: are smaller worlds easier to synchronize?, 2003 Phys. Rev. Lett. 91014101

[36] Reijneveld J C, Ponten S C, Berendse H W and Stam C J, The application of graph theoretical analysis to complex networks in the brain, 2007 Clin. Neurophysiol. 1182317

[37] Restrepo J G, Ott E and Hunt B R, Onset of synchronization in large networks of coupled oscillators, 2005 Phys. Rev. E 71036151 
The correlation of metrics in complex networks with applications in functional brain networks

[38] Restrepo J G, Ott E and Hunt B R, Approximating the largest eigenvalue of network adjacency matrices, 2007 Phys. Rev. E 76056119

[39] Rubinov M, Knock S A, Stam C J, Micheloyannis S, Harris A W, Williams L M and Breakspear M, Small-world properties of nonlinear brain activity in schizophrenia, 2009 Hum. Brain Mapp. 30403

[40] Rubinov M and Sporns O, Complex network measures of brain connectivity: uses and interpretations, 2010 Neuroimage $\mathbf{5 2} 1059$

[41] Simpson S L, Hayasaka S and Laurienti P J, Exponential random graph modeling for complex brain network, 2011 PLoS One 6 e20039

[42] Stam C J and van Dijk B W, Synchronization likelihood: an unbiased measure of generalized synchronization in multivariate data sets, 2002 Physica D 163236

[43] Stam C J, Jones B F, Nolte G, Breakspear M and Scheltens Ph, Small-world networks and functional connectivity in Alzheimer's disease, 2007 Cerebral Cortex 1792

[44] Stam C J and Reijneveld J C, Graph theoretical analysis of complex networks in the brain, 2007 Nonlinear Biomed. Phys. 13

[45] Van den Heuvel M P, Stam C J, Kahn R S and Hulshoff Pol H E, Efficiency of functional brain networks and intellectual performance, 2009 J. Neurosci. 297620

[46] Van Mieghem P, 2006 Performance Analysis of Communications Systems and Networks (Cambridge: Cambridge University Press)

[47] Van Mieghem P, Omic J and Kooij R E, Virus spread in networks, 2009 IEEE/ACM Trans. Netw. 171

[48] Van Mieghem P, Wang H, Ge X, Tang S and Kuipers F A, Influence of assortativity and degree-preserving rewiring on the spectra of networks, 2010 Eur. Phys. J. B 76643

[49] Van Mieghem P, 2011 Graph Spectra for Complex Networks (Cambridge: Cambridge University Press)

[50] van Wijk B C M, Stam C J and Daffertshofer A, Comparing brain networks of different size and connectivity density using graph theory, 2010 PLoS ONE 5 e13701

[51] Watanabe T and Masuda N, Enhancing the spectral gap of networks by node removal, 2010 Phys. Rev. E 82046102

[52] Watts D J and Strogatz S H, Collective dynamics of 'small-world' networks, 1998 Nature 393440 